\title{
Incidentes de fauna silvestre en líneas de energía en zona rural del Valle del Cauca, Colombia
}

\author{
Wildlife killing by power lines in rural areas of the \\ Valle del Cauca, Colombia
}

\author{
Carlos A. Saavedra-Rodríguez ${ }^{1,2}$, Álvaro Lizcano ${ }^{3}$, Juan David Corrales ${ }^{2}$
}

Resumen

\begin{abstract}
Introducción: Las líneas de energía causan electrocuciones de mamíferos arborícolas, representan una amenaza para la conservación local de estas especies. Objetivo: Documentar la electrocuciones de fauna silvestre en líneas de energía en zonas rurales de la ciudad de Cali, departamento del Valle del Cauca, Colombia. Metodología: se recopiló la información suministrada por los trabajadores de la empresa que realiza el mantenimiento de las líneas de energía, Megaproyectos SA. Resultados: Obtuvimos reporte de electrocuciones de Caluromys derbianus y Aotus lemurinus. Conclusiones: Los reportes resaltan la necesidad de evaluar la amenaza que las líneas de energía significan para las poblaciones de especies de fauna arborícola y de generar una normativa electrotécnica para prevenir las electrocuciones.
\end{abstract}

Palabras clave: Aotus lemurinus, Caluromys derbianus, Colombia, Electrocuciones, Líneas de energía, Valle del Cauca.

\begin{abstract}
Introduction: The energy lines cause electrocutions of arboreal mammals. These are threats for the local conservation of these species. Objective: To document electrocutions of wild fauna in energy lines occurred in rural zones of Cali municipality, department of Valle del Cauca, Colombia. Methodology: We obtained data from the workers of company that realized the energy lines maintaining, Megaproyectos SA. Results: We reported electrocutions of Caluromys derbianus and Aotus lemurinus. Conclusions: These reports provided information about the species presence and abundance in Andes of Colombia and highlighted the need of make risk evaluations of the energy lines over animal population and to generate technical laws for the prevention of the fauna killing.
\end{abstract}

Keywords: Aotus lemurinus, Caluromys derbianus, Colombia, Electrocutions, Electricity lines, Valle del Cauca.

\section{Introducción}

Las carreteras, líneas de energía y embalses son perturbaciones que afectan directamente a la fauna silvestre. Los estudios documentan el efecto sobre individuos ( Petrucci et al. 2009; Rowden et al. 2008), poblaciones (Ferrer y Negro 1992) y las medidas co- rrectoras implementadas tendientes a minimizar las mortalidades (Negro et al. 1989; Fernie y Bird 2000; Kaluga et al. 2011). En el neotrópico, el tema es relativamente reciente y en Colombia, hay algunos estudios que abordan esta temática (De La Zerda y Rosselli 2003; Quintero-Ángel et al. 2012; VargasSalinas et al. 2011).

\footnotetext{
Grupo de Investigación en Ecología Animal, Departamento de Biología, Universidad del Valle, Cali, Colombia. Estudiante de doctorado en Ciencias Biología de la Universidad del Valle financiado por COLCIENCIAS. e-mail: casaavedrar@yahoo.com Wildlife Conservation Society Colombia Program, Cali, Colombia.

3 Empresa Mega Proyectos de Iluminaciones de Colombia SA, Cali, Colombia. e-mail: lizcano.alvaro@gmail.com Fecha recepción: Junio 23, 2013 Fecha aprobación: Noviembre 7, 2013 Editor asociado: Mantilla H.
} 
La mortalidad de fauna silvestre en líneas de energía tiene connotación especial en mamíferos arborícolas porque estos usan las líneas de energía para desplazarse, por lo que es frecuente la muerte de los individuos a causa de electrocuciones. Estos eventos se han documentado para los colobos angoleños (Colobus angolensis) en África Central (Global Spirit 2010), los monos ardilla (Saimiri oerstedii) en Centroamérica (Boinski et al. 1998), los monos aulladores marrones (Alouatta guariba) (Petrucci et al. 2009) y las chuchas lanudas occidentales (Caluromys lanatus) en Suramérica (Martinelli 2010). Por tanto, las líneas de energía son amenazas no naturales y de preocupación para la conservación de mamíferos arborícolas amenazados. Para evitar estas electrocuciones se emplean técnicas relativamente sencillas pero eficaces, como es el aislar los conductores (Fernández y Azcona 2002; De La Zerda y Rosselli 2003).

En la presente nota se documenta las electrocuciones de especímenes de Caluromys derbianus y Aotus lemurinus encontrados en líneas de energía de zonas rurales de la ciudad de Cali, departamento del Valle del Cauca, Colombia. La información ha sido suministrada por la empresa Mega Proyectos de Iluminaciones de Colombia SA.

Caluromys derbianus o zarigüeya lanuda centroamericana, se conoce en Colombia como chucha lanuda (Rodríguez-Mahecha et al. 1995). C. derbianus habita bosques y es predominantemente arbórea (Emmons y Feer 1997). Fue considerada vulnerable por la Unión Internacional para la Conservación de la Naturaleza (IUCN) en 1996, actualmente se considera de menor preocupación (Lew et al. 2008) y algunas poblaciones parecen estar decreciendo debido a la pérdida de bosques. En Colombia no se considera amenazada pero en el Valle del Cauca, al suroccidente del país, hay incertidumbre sobre el estado de sus poblaciones (González-Anaya y CastilloCrespo 2007).

Aotus lemurinus se conoce en Colombia como mono nocturno marteja (Rodríguez-Mahecha et al. 1995). A. lemorinus habita bosques primarios, secundarios y fragmentos de bosques de niebla (Castaño y Cardona 2005), donde se encuentran más activos al amanecer y al atardecer. La especie está clasificada como vulnerable a escala global (MoralesJiménez y De La Torre 2008) y nacional (Rodríguez-
Mahecha et al. 2006), y se considera en peligro en el Valle del Cauca (Crespo-Castillo y González-Anaya 2007).

Durante los recorridos regulares que realizan los empleados de la empresa de mantenimiento Mega Proyectos de Iluminaciones de Colombia SA, entre junio y septiembre de 2012 se encontraron dos individuos de chucha lanuda en el sistema de líneas de energía dispuesto en la zona rural de la ciudad de Cali, Valle del Cauca, Colombia (Figura 1). Uno, en la vereda San Antonio, Corregimiento del Saladito, municipio de Felidia, Valle del Cauca, a 1800 m, y otro ejemplar encontrado en el corregimientos de la Buitrera, municipio de Cali, Valle del Cauca, a 1300 $\mathrm{m}$ (Figura2).

Se encontraron dos individuos de mono nocturno entre junio 2010 y abril 2011. El primero, en junio 2010 , se encontró en un poste del alumbrado en el corregimiento de Pichindé, vía a La Leonera. Se encontró el cráneo y huesos; su cola estaba envuelta al brazo galvanizado de la luminaria y una de sus extremidades anteriores estaba agarrada al conector; del ejemplar se conserva el cráneo. El segundo ejemplar fue encontrado en un poste de alumbrado público en el corregimiento El Saladito, sector San Antonio, finca Los Guácharos. El ejemplar preservaba la piel en buen estado, lo que indica que no llevaba mucho tiempo desde la electrocución (Figura2). El ejemplar estaba aferrado a las dos fases a la altura de los conectores metálicos, lo que le provocó una descarga de 220 voltios; esta es la carga de las líneas de alumbrado público.

Los especímenes hallados electrocutados son útiles en diferentes marcos de estudio (Gottdenker et al. 2001). Para Caluromys, que no es común en colecciones, el reporte es importante para determinar su ocurrencia y brinda unos primeros indicios de abundancia. Los individuos de chucha lanuda y de mono nocturno son importantes en el marco de la conservación de la biodiversidad regional. Los hallazgos en zonas rurales, que han sido fuertemente modificadas pero que mantienen vegetación nativa típica y zonas de jardines, resalta la importancia de estas áreas para albergar mamíferos arborícolas y de la necesidad de orientar políticas de manejo. Siendo la chucha lanuda y el mono nocturno especies amenazadas en la región, podrían ser objeto de seguimiento y monitoreo respecto a las mortalidades por electrocuciones y de 
las políticas de manejo que se implementen.

La empresa Mega Proyectos de Iluminaciones de Colombia SA encargada del mantenimiento de líneas de energía de algunas zonas rurales del Valle del Cauca inició acciones para aislar estas trampas electrificadas. La empresa ha determinado que en proyectos de expansión de alumbrado público a desarrollarse en la zona rurales, se usará solo cableado aislado y grapas de perforación. Estas mediadas junto con un monitoreo de las poblaciones contribuirían a evaluar el beneficio sobre las poblaciones y a identificar las mejores acciones para reducir los efectos sobre la fauna silvestre.

Para eliminar las electrocuciones en líneas de energía es necesario evaluar los riesgos de estas infraestructuras, aprobar normativa electrotécnica y realizar acciones que reduzcan la mortalidad (aislamientos de conductores, balizamiento, etc) en colaboración con las compañías eléctricas. En la selva keniata de Diani, por ejemplo, para evitar las electrocuciones de monos colobos angoleños (Colobus angolensis), organizaciones no gubernamentales han identificado los puntos geográficos que concentran la mayor parte de las electrocuciones y gracias a un convenio con la compañía eléctrica de Kenia, se ha comenzado el aislamiento de las líneas de energía (Global Spirit 2010). Esta alianza es un modelo que podría ser aplicado en Colombia, permitiendo acompañar a las empresas de energía en la gestión para manejar los conflictos y para proteger la fauna silvestre.

\section{Agradecimientos}

A los funcionarios de la empresa Mega Proyectos de Iluminaciones de Colombia SA por su colaboración en mantener los especímenes que encontraron electrocutados. A la empresa por permitirnos utilizar los datos obtenidos.

\section{Literatura citada}

Boinski S, Jack K, Lamarsh C, Coltrane JE. 1998.

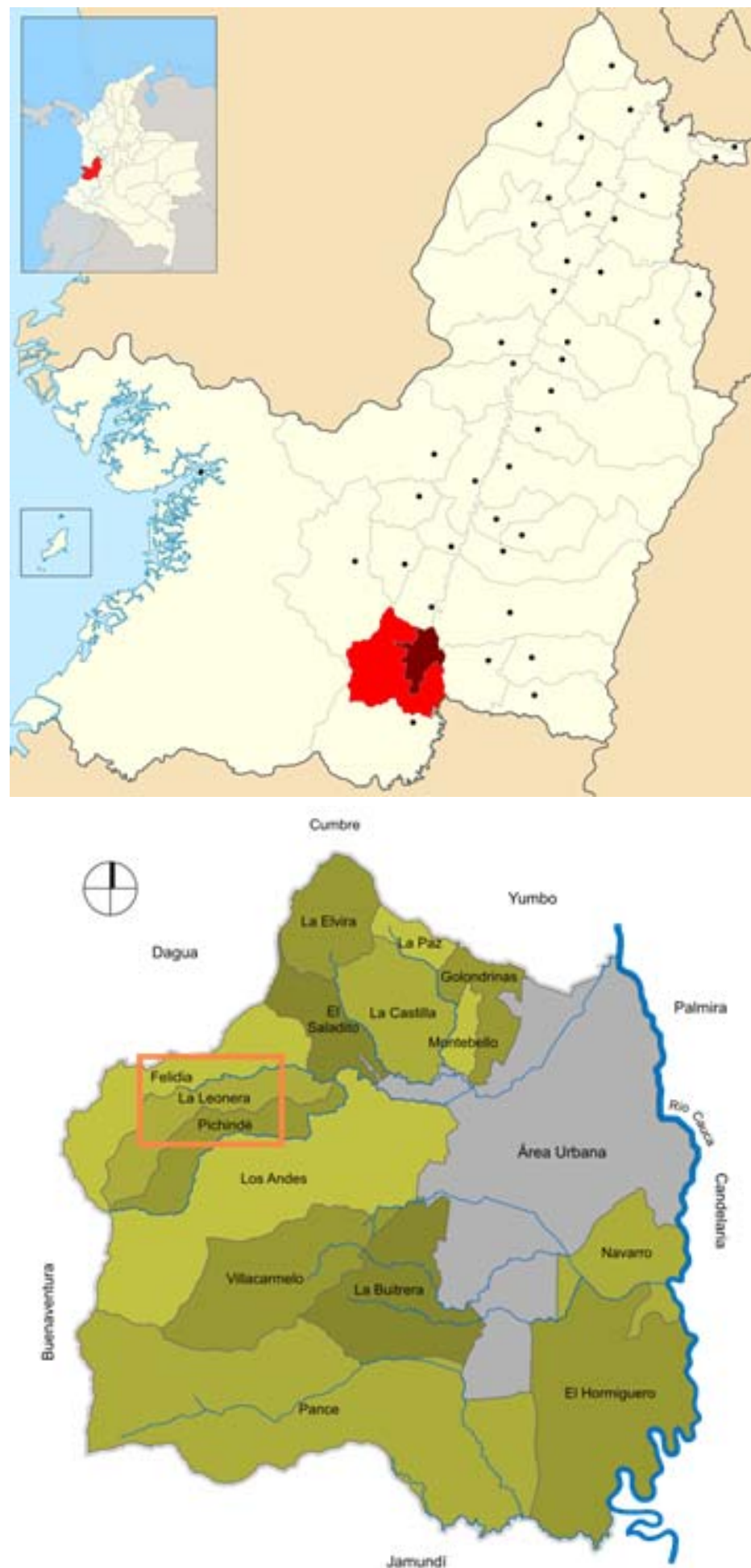

Figura 1. Area de hallazgo de especimenes de chuca lanuda (Caluromys derbianus) y marteja (Aotus lemurinus) electrocutados en líneas de energía en zona rural de la ciudad de Cali, departamento del Valle del Cauca.

Fuente de mapas: Wikipedia. 


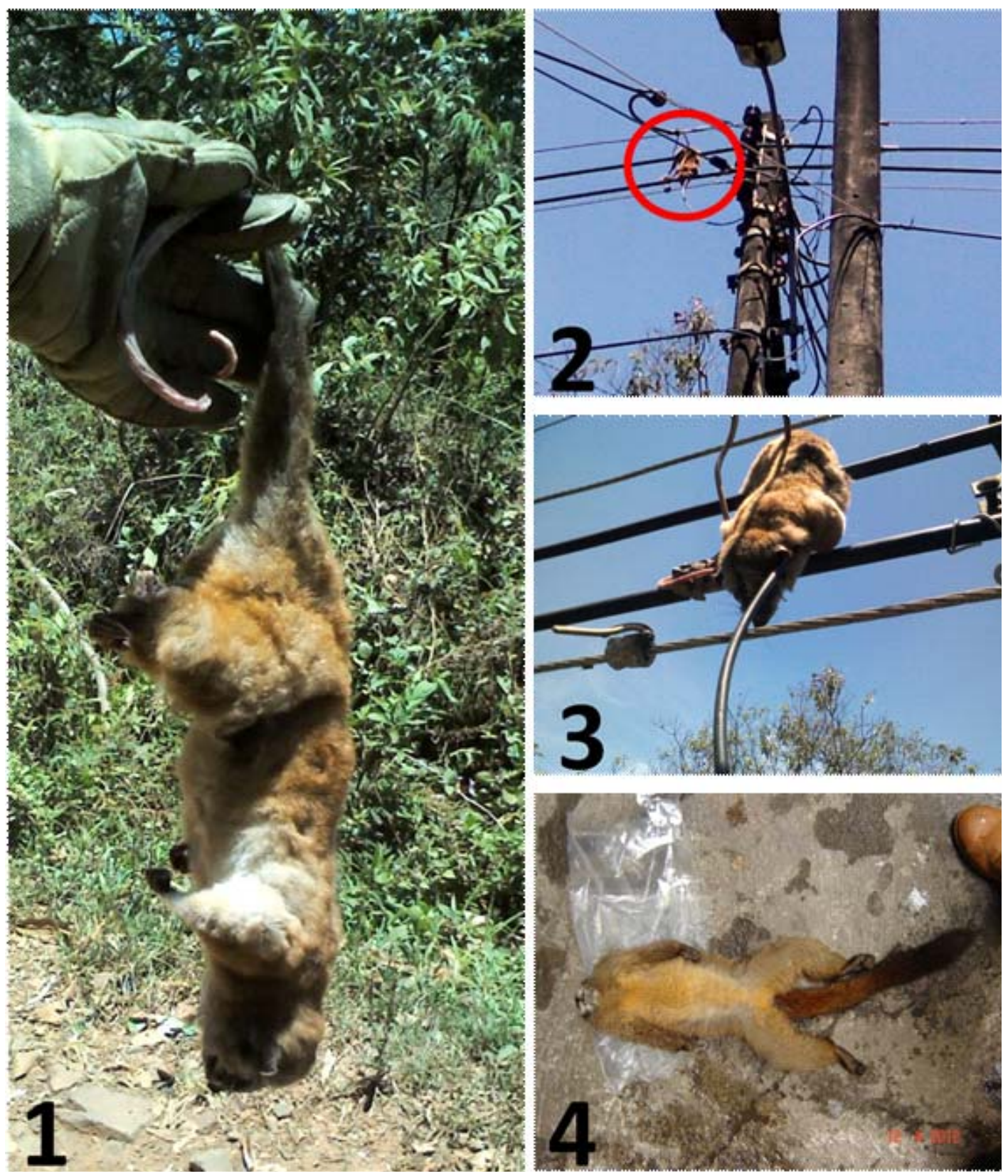

Figura 2. Ejemplares de chucha lanuda (Caluromys derbianus) (1-3) y de marteja (Aotus lemorinus) (4) encontrados electrocutados en líneas de energía en zona rural de la ciudad de Cali, departamento del Valle del Cauca.

Squirrel monkeys in Costa Rica: drifting to extinction. Oryx. 32: 45-58.

Castaño JH, Cardona DM. 2005. Presencia del mono nocturno andino (Aotus lemurinus I. Geoffroy-St. Hilaire, 1843) en fragmentos de bosque de la cuenca media del río Cauca. Bol Cient Mus Hist Nat Univ Caldas. 9: 111-7.

Castillo-Crespo LS, González-Anaya M. 2007. Avances en la implementación del Plan de Acción en Biodiversidad del Valle del Cauca. Dirección Técnica Ambiental, Grupo Biodiversidad. Cali: Corporación Autónoma Regional del Valle del Cauca, CVC. 66 pp.

De La Zarna S, Rosselli L. 2003. Mitigación de colisión de aves contra líneas de transmisión eléctrica con marcaje del cable de guarda. Ornitol Colomb. 1: 42-62.

Emmons LH, Feer F. 1997. Neotropical rainforest mammals: $a$ field guide. Chicago: University of Chicago Press. $307 \mathrm{pp}$.

Fernández C, Azcona P. 2002. Tendidos eléctricos y medio ambiente en Navarra. Pamplona: Ed. Gobierno de Navarra. Medio Ambiente.

Fernie KJ, Bird DM. 2000. Effects of electromagnetic fields on the growth of nestlings American Kestrels. Condor. 102: 461-5.

Ferrer M, Negro JJ. 1992. Tendidos eléctricos y conservación de aves en España. Ardeola. 36: 23-7. 
Global Spirit. 2010. Project Colobus monkeys in Kenya. Extensive information. URL disponible en: http://www.global spirit.nl/web/pages/en/GS12CAKE.html $28 \mathrm{pp}$.

Gottdenker N, Wallace RB, Gómez H. 2001. La importancia de los atropellos para la ecología y conservación: Dinomys branickii un ejemplo de Bolivia. Ecol Bolivia. 35: 61-7.

Kaluga I, Sparks TH, Tryjanowski P. 2011. Reducing death by electrocution of the white stork Ciconia ciconia. Cons Letters. 4: 483-7.

Lew D, Soriano P, Cuarón AD, Emmons L, Reid F, Helgen K. 2008. Caluromys derbianus. En: UICN 2012. Lista Roja de la UICN de Especies Amenazadas. Versión 2012.2. (fecha de acceso enero 20 de 2013) URL disponible en: www.iucnredlist.org.

Martinelli AG. 2010. Nuevo registro de la cuica lanosa, Caluromys lanatus (Olfers, 1818) (Mammalia, Didelphimorphia, Didelphidae) en el oeste del estado de Minas Gerais, Brasil. Not Faunist. 59:1-3.

Morales-Jiménez AL, De La Torre S. 2008. Aotus lemurinus. En: IUCN 2012. IUCN Red List of Threatened Species. Version 2012.2. (fecha de acceso enero 23 de 2013). URL disponible en: www.iucnredlist.org

Negro JJ, Ferrer M, Santos C, Regjdor S. 1989. Eficacia de dos métodos para prevenir electrocuciones de aves en tendidos eléctricos. Ardeola. 36:201-6.
Petrucci MP, Pontes LAE, Queiroz FF. 2009. Electrocution accident in free-ranging bugio (Alouatta fusca) with subsequent amputation of the forelimb: case report. Rev Port Cien Vet. 104:87-90.

Rodríguez-Mahecha JV, Hernández-Camacho JI, Defler T. 1995. Mamíferos colombianos: sus nombres comunes e indígenas. Occasional Papers in Conservation Biology. Conservat Internal. 3: 1-56.

Rodríguez-Mahecha JV, Alberico M, Trujillo F, Jorgenson J (eds.) 2006. Libro Rojo de los Mamíferos de Colombia. Serie Libros Rojos de Especies Amenazadas de Colombia. Bogotá: Conservación Internacional Colombia, Ministerio de Ambiente, Vivienda y Desarrollo Territorial.429 pp.

Rowden PJ, Steinhardt ADA, Sheehan MC. 2008. Road crashes involving animals in Australia. Accid Anal Prevent. 40:1865-71.

Quintero-Ángel A, Osorio-Domínguez D, Vargas-Salinas F, Saavedra-Rodríguez CA. 2012. Roadkill rate of snakes in a disturbed landscape of Central Andes of Colombia. Herpetol Notes. 5: 99-105.

Vargas-Salinas F, Delgado-Ospina I, López-Aranda F. 2011. Mortalidad por atropello vehicular y distribución de anfibios y reptiles en un bosque subandino en el occidente de Colombia. Caldasia. 33:121-38. 